Correspondence

Franz-Christoph Bange

bange.franz@mh-hannover.de

Received 13 August 2008

Revised 17 December 2008

Accepted 22 December 2008

\title{
The roles of the nitrate reductase NarGHJl, the nitrite reductase NirBD and the response regulator GlnR in nitrate assimilation of Mycobacterium tuberculosis
} Insa Joost, ${ }^{1}$ Isabel Weber, ${ }^{1}$ Sarah Horst, ${ }^{1}$ Birgit Ackermann, ${ }^{1}$ Mascha Schmidt, ${ }^{1}$ Wolfgang Wohlleben, ${ }^{2}$ Stefan Ehlers, ${ }^{4}$ Robert Geffers, ${ }^{3}$ Jens Reuther ${ }^{2}$ and Franz-Christoph Bange ${ }^{1}$

\footnotetext{
${ }^{1}$ Department of Medical Microbiology and Hospital Epidemiology, Medical School Hannover, Carl-Neuberg-Strasse 1, 30625 Hannover, Germany

${ }^{2}$ Microbiology/Biotechnology, Microbiological Institute, Faculty of Biology, Eberhard-KarlsUniversität Tübingen, Auf der Morgenstelle 28, 72076 Tübingen, Germany

${ }^{3}$ Department of Cell Biology, Helmholtz Centre for Infection Research, Inhoffenstrasse 7, 38124 Braunschweig, Germany

${ }^{4}$ Molecular Infection Biology, Research Center Borstel, Leibniz-Center for Medicine and Biosciences, 23845 Borstel, Germany
}

\begin{abstract}
Mycobacterium tuberculosis can utilize various nutrients including nitrate as a source of nitrogen. Assimilation of nitrate requires the reduction of nitrate via nitrite to ammonium, which is then incorporated into metabolic pathways. This study was undertaken to define the molecular mechanism of nitrate assimilation in $M$. tuberculosis. Homologues to a narGHJ/-encoded nitrate reductase and a nirBD-encoded nitrite reductase have been found on the chromosome of $M$. tuberculosis. Previous studies have implied a role for NarGHJ in nitrate respiration rather than nitrate assimilation. Here, we show that a narG mutant of $M$. tuberculosis failed to grow on nitrate. A nirB mutant of $M$. tuberculosis failed to grow on both nitrate and nitrite. Mutant strains of Mycobacterium smegmatis $\mathrm{mc}^{2} 155$ that are unable to grow on nitrate were isolated. The mutants were rescued by screening a cosmid library from $M$. tuberculosis, and a gene with homology to the response regulator gene $g \ln R$ of Streptomyces coelicolor was identified. A $\Delta g \ln R$ mutant of M. tuberculosis was generated, which also failed to grow on nitrate, but regained its ability to utilize nitrate when nirBD was expressed from a plasmid, suggesting a role of $\mathrm{G} \ln \mathrm{R}$ in regulating nirBD expression. A specific binding site for $\mathrm{G} \operatorname{lnR}$ within the nirB promoter was identified and confirmed by electrophoretic mobility shift assay using purified recombinant GlnR.

Semiquantitative reverse transcription $\mathrm{PCR}$, as well as microarray analysis, demonstrated upregulation of nirBD expression in response to GlnR under nitrogen-limiting conditions. In summary, we conclude that NarGHJ and NirBD of $M$. tuberculosis mediate the assimilatory reduction of nitrate and nitrite, respectively, and that $\mathrm{G} \ln \mathrm{R}$ acts as a transcriptional activator of nirBD.
\end{abstract}

\section{INTRODUCTION}

Mycobacterium tuberculosis has only limited access to nutrients in infected tissue (Munoz-Elias \& McKinney, 2006). Nitrate, however, is available in infected tissue, as it

Abbreviation: EMSA, electrophoretic mobility shift assay.

The GEO database accession number for the microarray dataset associated with this paper is GSE13246. is generated spontaneously from nitric oxide (NO), the product of nitric oxide synthase (Bogdan, 2001). Assimilation of nitrogen into mycobacterial metabolism is essential for the survival of M. tuberculosis in vitro and in vivo. Assimilation of nitrate by $M$. tuberculosis was reported more than 40 years ago (DeTurk \& Bernheim, 1958; Hedgecock \& Costello, 1962; Virtanen, 1960). However, its molecular basis remains unknown. 
The first step in nitrate assimilation is the reduction of nitrate $\left(\mathrm{NO}_{3}^{-}\right)$to nitrite $\left(\mathrm{NO}_{3}^{-}\right)$. In Bacillus subtilis, assimilatory nitrate reduction is mediated by a distinct cytoplasmic enzyme. A different nitrate reductase, encoded by narGHJI, is membrane bound and serves respiratory functions. NarJ assembles the subunits NarG, $\mathrm{H}$ and I to the functional nitrate reductase. In B. subtilis, its expression is typically induced under anaerobic conditions (Gennis \& Stewart, 1996; Nakano \& Zuber, 1998; Ogawa et al., 1995). Analysis of the genome of $M$. tuberculosis revealed genes with $30 \%$ and $50 \%$ homology at the amino acid level to those found in the NarGHJI of B. subtilis. We previously reported that NarGHJI of $M$. tuberculosis mediates reduction of nitrate, not only under anaerobic, but also under aerobic conditions (Stermann et al., 2004; Weber et al., 2000). This is in accordance with data from Sohaskey \& Wayne (2003), who reported that expression of narGHJI was not dependent on anaerobiosis. As genes with homology to a distinct assimilatory nitrate reductase were not identified in the genome of $M$. tuberculosis, the initial goal of this study was to examine whether NarGHJI of $M$. tuberculosis has an assimilatory function.

The second step in nitrate assimilation is reduction of nitrite $\left(\mathrm{NO}_{3}^{-}\right)$to ammonium $\left(\mathrm{NH}_{4}^{+}\right)$. The genome of $M$. tuberculosis revealed genes with $40 \%$ and $50 \%$ homology, at the amino acid level, to nirBD of Escherichia coli (Cole et al., 1998). This operon encodes a sirohaem-dependent $\mathrm{NADH}$-nitrite reductase, an enzyme that typically mediates nitrate assimilation in various bacteria and fungi (Lin \& Stewart, 1998). Only in E. coli and in other enterobacteria is nirBD-encoded nitrite reductase enzyme induced under anaerobic conditions, and it does not function as an assimilatory nitrite reductase but detoxifies nitrite that accumulates from nitrate respiration (Gennis \& Stewart, 1996). We included analysis of nirB of M. tuberculosis with respect to its assimilatory function in this study.

Regulation of nitrate assimilation may be subject to a general nitrogen regulation (ntr) system, which depends on the presence of the preferred nitrogen source. It might also be pathway specific, and is then controlled by the availability of nitrate. In two actinobacteria, Corynebacterium and Streptomyces, the transcriptional regulation of nitrogen assimilation has been analysed. In Corynebacterium glutamicum, the regulator protein AmtR is responsible for this process (Beckers et al., 2005; Burkovski, 2007). In Streptomyces coelicolor, GlnR controls regulation of genes involved in nitrogen metabolism (Fink et al., 2002; Tiffert et al., 2008; Wray et al., 1991). Regulation of nitrate assimilation has not been specifically addressed in either species, or in M. tuberculosis.

In the present study, assimilation of nitrate was measured as growth on nitrate as a sole source of nitrogen. We found robust growth of $M$. tuberculosis wild-type, whereas neither a narG mutant nor a nirB mutant grew under these conditions, suggesting assimilatory functions for both genes. We also identified $\mathrm{GlnR}$ as a regulator for expression of the nirBD-encoded assimilatory nitrite reductase.

\section{METHODS}

Strains and cultures. Mycobacterium tuberculosis $\mathrm{H} 37 \mathrm{Rv}$ and Mycobacterium smegmatis $\mathrm{mc}^{2} 155$ (Snapper et al., 1990) were cultured in 7H9 broth or on 7H10 plates (Difco) supplemented with $0.2 \%$ glycerol, $0.05 \%$ Tween 80 for all liquid media, and $10 \%$ ADS ( $0.5 \%$ Bovine Albumin Fraction V, $0.2 \%$ glucose, $140 \mathrm{mM} \mathrm{NaCl}$ ) unless indicated otherwise. Assimilation of nitrate or nitrite was tested under nitrogen-limiting conditions using a basal medium, if necessary solidified with $1.5 \%$ agar, supplemented with $\mathrm{KNO}_{3}, \mathrm{KNO}_{2}$ or $\mathrm{NH}_{4} \mathrm{Cl}$ as sole source of nitrogen, $0.5 \mathrm{mM} \mathrm{MgCl}_{2}, 0.5 \mathrm{mM} \mathrm{CaCl}$, $10 \%$ ADS, $0.2 \%$ glycerol and $0.05 \%$ Tween 80 ( 11 of the basal medium contained $1 \mathrm{~g} \mathrm{KH}_{2} \mathrm{PO}_{4}, 2.5 \mathrm{~g} \mathrm{Na}_{2} \mathrm{HPO}_{4}, 2 \mathrm{~g} \mathrm{~K}_{2} \mathrm{SO}_{4}$ and $2 \mathrm{ml}$ of trace elements; 11 of trace elements contained $40 \mathrm{mg} \mathrm{ZnCl}_{2}$, $200 \mathrm{mg} \mathrm{FeCl} \cdot 6 \mathrm{H}_{2} \mathrm{O}, 10 \mathrm{mg} \mathrm{CuCl} 2 \cdot 4 \mathrm{H}_{2} \mathrm{O}, 10 \mathrm{mg} \mathrm{MnCl} \cdot 4 \mathrm{H}_{2} \mathrm{O}, 10 \mathrm{mg}$ $\mathrm{Na}_{2} \mathrm{~B}_{4} \mathrm{O}_{7} \cdot 10 \mathrm{H}_{2} \mathrm{O}$ and $\left.10 \mathrm{mg}\left(\mathrm{NH}_{4}\right)_{6} \mathrm{Mo}_{7} \mathrm{O}_{24} \cdot 4 \mathrm{H}_{2} \mathrm{O}\right)$. To test for accumulation of nitrite, as a nitrogen-limiting medium a modified Proskauer Beck was used that contained, per litre, $5 \mathrm{~g} \mathrm{KH}_{2} \mathrm{PO}_{4}, 0.6 \mathrm{~g}$ $\mathrm{MgSO}_{4} .7 \mathrm{H}_{2} \mathrm{O}, 2.5$ g C$_{6} \mathrm{H}_{6} \mathrm{MgO}_{7}, 15 \mathrm{ml}$ glycerol, $2.5 \mathrm{ml} 20 \%$ Tween 80 and $10 \mathrm{mM}$ nitrate.

Generation of mutants in $\boldsymbol{M}$. tuberculosis. Construction of the narG mutation in $M$. tuberculosis has been described previously (Stermann et al., 2003). Cosmids from a genomic library of $M$. tuberculosis (Bange et al., 1999) carrying nirB or $g \ln R$ were obtained by colony hybridization. A $1352 \mathrm{bp}$ fragment and a $323 \mathrm{bp}$ fragment were deleted in $\operatorname{nir} B$ and $g \ln R$, respectively, and appropriate fragments were cloned into the PacI site of the previously described pYUB657 (Pavelka \& Jacobs, 1999). M. tuberculosis was transformed and screened for clones resistant to hygromycin. Sucrose was used for counter-selection, as described previously, to select clones in which double crossovers had occurred (Pavelka \& Jacobs, 1999).

For complementation experiments, an $8652 \mathrm{bp}$ EcoRV fragment containing the narGHJI gene cluster, a $3959 \mathrm{bp} M l u \mathrm{I}$ fragment containing the nirBD gene cluster, and a 1582 bp EcoRV-SmaI fragment containing $g \ln R$ were chosen. The fragments were subcloned from cosmids into the promoterless pMV306 vector. In addition, the 1087 bp HindIII-BamHI fragment containing $g \ln R$ of $S$. coelicolor was inserted into the HpaI site of pMV261.

Testing for growth on nitrate, nitrite or ammonium. Bacteria were cultured in $7 \mathrm{H} 9$ broth to an $\mathrm{OD}_{600}$ between 0.7 and 1.0. The cultures were washed with nitrogen-limiting medium before the $\mathrm{OD}_{600}$ was adjusted to 0.1 . Cultures were incubated at $37^{\circ} \mathrm{C}$ with good aeration. Medium was supplemented with $10 \mathrm{mM}$ nitrate, $1 \mathrm{mM}$ nitrite or $10 \mathrm{mM}$ ammonium chloride and growth was tested by measuring the $\mathrm{OD}_{600}$.

Isolation and complementation of $\boldsymbol{M}$. smegmatis mutants, and mapping of the corresponding mutations. Ethyl methane sulphonate-mutagenized $M$. smegmatis $\mathrm{mc}^{2} 155$ clones (McKinney et al., 2000) were cultured on nitrogen-limiting agar containing either no nitrate or $10 \mathrm{mM} \mathrm{KNO}$, or on fully supplemented $7 \mathrm{H} 10$ plates. Two M. smegmatis strains, \#2009 and \#5192, which were unable to grow with $10 \mathrm{mM} \mathrm{KNO}_{3}$, were transformed with an integrating cosmid library of $M$. tuberculosis H37Rv. Cosmid pIW32 was recovered from a complemented $M$. smegmatis clone. From this cosmid, a NotI fragment containing Rv0816c to Rv0820 was subcloned into the promoterless integrating mycobacterial shuttle vector pMV306 (Stover et al., 1992), resulting in the construct pBA4. This construct was further subcloned into the construct pBA7 using EcoRV, and into pBA8 using NruI and PvuI. pBA7 and pBA8 contained only Rv0818 and its own promoter.

Accumulation of nitrite. Bacteria were adjusted to an $\mathrm{OD}_{600}$ of 0.2 in nitrogen-limiting medium containing $10 \mathrm{mM} \mathrm{KNO}_{3}$ and incu- 
bated at $37{ }^{\circ} \mathrm{C}$ with good aeration. At indicated time points, $100 \mu \mathrm{l}$ sulfanilic acid and $100 \mu \mathrm{l} N, N$-dimethyl-1-naphthylamine (API system, bioMérieux) was added to $1 \mathrm{ml}$ culture. Absorbance of the supernatant was measured at $440 \mathrm{~nm}$ and quantified, relating the values to a standard curve of nitrite.

\section{Expression of nirBD under the control of the hsp60 promoter in} the $\mathbf{g} \operatorname{In} \boldsymbol{R} \mathbf{M}$. tuberculosis mutant. The constitutive hsp60 promoter from pMV261 was fused to nirBD. For the initial PCR, 261Fus\#1 (5'TGTGGTGGCATCCGTGGC-3') and 261Fus\#2 (5'-CGCGAACTCCCAGCCGTAGGCATTGCGAAGTGATTCCTCC-3') were used, whereas the second PCR was performed with nirBFus\#1 (5'-TCCGGAGGAATCACTTCGCAATGCCTACGGCTGGGAGTTCG-3') and nirBFus\#2 (5'-GACTCGATCGACTCGGTACCG-3'). PCR products were used as a template for a self-primed PCR with the terminal primer pair 261Fus\#1 and nirBFus\#2. This fusion molecule was cut with $K p n \mathrm{I}$ and cloned via the $K p n \mathrm{I}$ sites into the promoterless, integrating mycobacterial plasmid pMV306 containing nirBD on a $M l u I$ fragment. This construct was transformed into the $g \ln R$ mutant. Growth curves were obtained by culturing the respective strains as described above.

Preparation of RNA from $\boldsymbol{M}$. tuberculosis. $\Delta g \ln R$ M. tuberculosis and the wild-type strain were cultured in 7H9 to the mid-exponential growth phase. Then, the cells were washed with nitrogen-limiting medium supplemented with $5 \mathrm{mM} \mathrm{KNO}_{3}$ and incubated for $18 \mathrm{~h}$ or $48 \mathrm{~h}$ before RNA was extracted. Cultures were incubated with an equal volume of GTC buffer [ $5 \mathrm{M}$ guanidinium thiocyanate, $0.5 \%$ $(\mathrm{w} / \mathrm{v}) \quad \mathrm{n}$-laurylsarcosine, $0.7 \% \quad(\mathrm{w} / \mathrm{v})$ sodium citrate, $0.7 \% \quad \beta$ mercaptoethanol], centrifuged and resuspended in $1 \mathrm{ml}$ Trizol reagent (Invitrogen). The cells were disrupted and extracted once with $\mathrm{CHCl}_{3}$, and re-extracted using the RNeasy Mini-kit (Qiagen). The optional DNase I on-column digest was extended to $1 \mathrm{~h}$ and an additional DNase I digest (NEB) was performed with the eluate for $45 \mathrm{~min}$. After that, RNA was purified again with the RNeasy Mini-kit.

RT-PCR of RNA from $\boldsymbol{M}$. tuberculosis. RNA was extracted after incubation of bacteria in nitrogen-limiting medium for $18 \mathrm{~h}$. cDNA, which was generated by random priming of $2 \mu \mathrm{g}$ RNA, was diluted $1: 20$ and $1: 5$. PCRs were performed with $2 \mu \mathrm{M}$ of each of the following primers: qrt_sigA_mtu3 (as control) (5' -CACGCAAGGACGCCGAACTC-3'), qrt_sigA_mtu4 (as control) (5' -TACAGGCCAGCCTCGATCCG-3'), qrt_nirB_mtu3 (5'-GTCCCGGTTCGTTTCCTTCG-3') and qrt_nirB_mtu4 (5'-CGCGGGATACCAATGGACAC$\left.3^{\prime}\right)$. The PCR conditions were $95{ }^{\circ} \mathrm{C}$ for $5 \mathrm{~min}$; then 35 cycles of $95{ }^{\circ} \mathrm{C}$ for $15 \mathrm{~s}, 55^{\circ} \mathrm{C}$ for $30 \mathrm{~s}$ and $72{ }^{\circ} \mathrm{C}$ for $20 \mathrm{~s}$; and finally $5 \mathrm{~min}$ at $72{ }^{\circ} \mathrm{C}$.

Expression of $\mathbf{g} \ln \boldsymbol{R}$ from $\boldsymbol{M}$. tuberculosis in E. coli. GlnR from $S$. coelicolor was expressed and purified as described previously (Tiffert et al., 2008). glnR of $M$. tuberculosis was amplified with the primers 5'-CATATGTTGGAGTTATTACTGCTGAC-3' and 5' -AAGCTTTCATTTTTCGAACTGCGGGTGGCTCCACTGACTGCGCAACGGGTC $-3^{\prime}$ by adding a sequence encoding a C-terminal StrepII-tag. The product was subcloned into pJOE2775, under the control of the $\mathrm{P}_{\text {rham }}$ promoter. Gene expression was induced with $0.2 \%$ rhamnose. Cells were harvested, washed with a solution of $50 \mathrm{mM}$ Tris, $150 \mathrm{mM} \mathrm{NaCl}$, $10 \mathrm{mM} \mathrm{MgCl}, 5 \%$ glycerol and $10 \mathrm{mM} \beta$-mercaptoethanol, $\mathrm{pH} 8$, and broken by French press (American Instruments). Then, Complete protease inhibitor cocktail (Roche) was added to the mixture. Cell debris and membrane fractions were removed by centrifugation (45 min; 15000 r.p.m.; $4{ }^{\circ} \mathrm{C}$ ). Purification of GlnR-Strep-tagged proteins from the soluble fraction was performed at $7{ }^{\circ} \mathrm{C}$ with StrepTactin Superflow gravity flow columns (IBA).

Electrophoretic mobility shift assay (EMSA). DNA fragments containing M. tuberculosis $\mathrm{H} 37 \mathrm{Rv}$ upstream regions were PCR- amplified using genomic DNA of M. tuberculosis $\mathrm{H} 37 \mathrm{Rv}$ as template. The primers $5^{\prime}$-AGCCAGTGGCGATAAGCCCACACCGGACGCGACCAC- $3^{\prime}$ and $5^{\prime}$-AGCCAGTGGCGATAAGGGTCTGAGGGTATGAGGGGC-3' for the upstream region of nirB were used. The underlined $5^{\prime}$-extensions have no homology to the template and were used for PCR labelling. The DNA fragments were purified using S-400 Microspins (GE Healthcare). Fragment labelling was performed via PCR using the Cy5-labelled primer 5'-AGCCAGTGGCGATAAG$3^{\prime}$.

Two nanograms of DNA was used in each EMSA reaction. For GlnR mixed with the nirB upstream region of M. tuberculosis, $16 \mu \mathrm{M}$ purified GlnR was used. The DNA and protein were incubated in a reaction buffer $(50 \mathrm{mM}$ Tris, $100 \mathrm{mM} \mathrm{NaCl}, 10 \mathrm{mM} \beta$-mercaptoethanol, $\mathrm{pH}$ 8) for $10 \mathrm{~min}$ at $24{ }^{\circ} \mathrm{C}$. The fragments were separated on $2 \%$ TAE agarose gels. DNA bands were visualized by fluorescence imaging using a Typhoon Trio + Variable Mode Imager (GE Healthcare).

Transcriptome analysis. $M$. tuberculosis wild-type and mutant were cultured in $7 \mathrm{H} 9$ to an $\mathrm{OD}_{600}$ of between 0.7 and 1.0. Bacteria were harvested by centrifugation and washed with nitrogen-limiting medium containing $5 \mathrm{mM} \mathrm{KNO}_{3}$. The $\mathrm{OD}_{600}$ was adjusted to approximately 0.7 with nitrogen-limiting medium supplemented with $5 \mathrm{mM} \mathrm{KNO}_{3}$ and cultures were incubated for about $18 \mathrm{~h}$ and $48 \mathrm{~h}$ at $37{ }^{\circ} \mathrm{C}$ with agitation before RNA was extracted as described above.

Equal amounts of RNA (between 3 and $10 \mu \mathrm{g}$ ) were subjected to reverse transcriptase reaction following the Affymetrix protocol. The cDNA was purified using the QIAquick PCR Purification. Custommade microarrays for $M$. tuberculosis $\mathrm{H} 37 \mathrm{Rv}$ were used for transcriptome analysis (Affymetrix). The chip contains 44033 probe pairs representing 4003 coding sequences, and 7902 probe pairs representing 1413 intergenic regions. Hybridization was done following the Affymetrix protocol. Microarrays were stained in the GeneChip Fluidics Station 450 according to the modified FlexMidi_euk2v3 program for Pseudomonas aeruginosa. Analysis of microarray data was performed using the Affymetrix GCOS 1.4 software. For normalization, all array experiments were scaled to a target intensity of 150, otherwise using the default values of GCOS 1.4. Signal intensities obtained from the individual measures for mutant $M$. tuberculosis and the wild-type strain at indicated time points were grouped and compared using $t$-test statistics. The entire dataset was submitted in MIAME-format to the GEO database (http://www.ncbi.nlm.nih.gov/projects/geo/), accession number GSE13246.

\section{RESULTS}

\section{Growth of $\Delta$ narG $M$. tuberculosis and $\Delta$ nirB $M$. tuberculosis on nitrate and nitrite}

Previously, we constructed a mutant of $M$. tuberculosis that carried a deletion in the narG gene (Stermann et al., 2003). Constitutive expression of narGHJI in M. tuberculosis has been reported, but the issue of whether the narGHJIencoded nitrate reductase provides assimilatory functions has not been addressed. Thus, we tested growth of the narG mutant in nitrogen-limiting medium, with nitrate as the sole nitrogen source. The mutant had lost the ability to assimilate nitrate. Introduction of narGHJI from $M$. tuberculosis into the mutant restored the wild-type phenotype (Fig. 1a). Growth of the $\Delta$ narG mutant on 

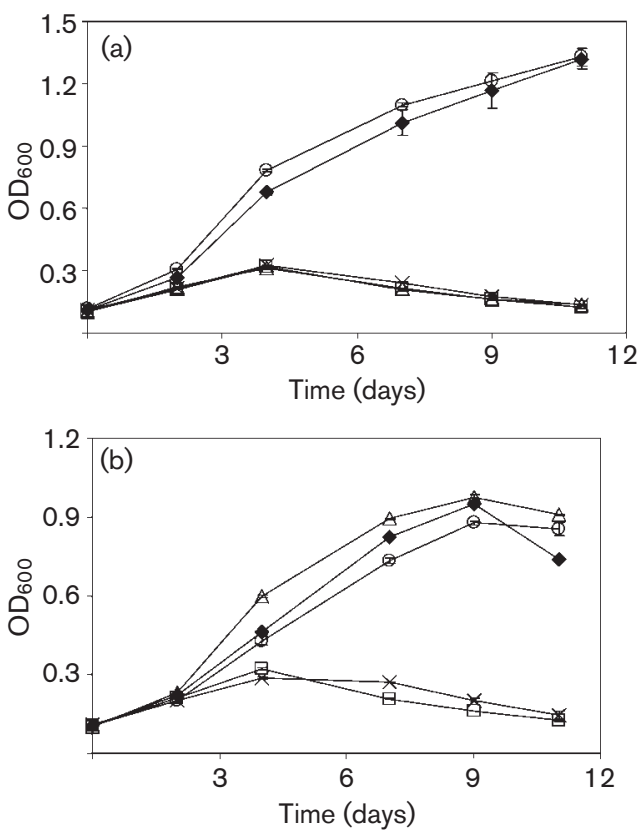

Fig. 1. Growth of $\Delta$ narG $M$. tuberculosis and $\Delta$ nirB $M$. tuberculosis on nitrate and nitrite. Bacteria were cultured in nitrogen-limiting medium containing (a) $10 \mathrm{mM}$ nitrate or (b) $1 \mathrm{mM}$ nitrite as the only nitrogen source. Aliquots were tested for growth by measuring $\mathrm{OD}_{600}$. Cultures were kept with good aeration. The following strains were compared: wild-type M. tuberculosis ( $)$, $\Delta$ narG M. tuberculosis $(\triangle)$ or $\Delta$ nirB M. tuberculosis $(\times), \Delta$ narG $M$. tuberculosis : : narGHJI ( $\bigcirc$ in a) and $\triangle$ nirB M. tuberculosis : : nirBD ( $\bigcirc$ in b). Wild-type M. tuberculosis was cultured in nitrogenlimiting medium without nitrate or nitrite as a control $(\square)$.

nitrite was unimpaired (Fig. 1b). These results suggest that narGHJI mediates nitrate assimilation in M. tuberculosis.

Nitrate assimilation requires the concerted action of a nitrate and nitrite reductase, followed by incorporation of ammonium into cellular metabolism via glutamine synthetase and glutamate synthase. Sequencing of $M$. tuberculosis revealed homology to only one nitrite reductase encoded by nirB and nirD (Rv0252, Rv0253) (Cole et al., 1998). As no functional studies have been conducted to date, we constructed a deletion mutant of nirB in M. tuberculosis to elucidate its role in the second step of assimilation of nitrate. A $1352 \mathrm{bp}$ fragment within nirB was deleted from the chromosome, and growth of $\Delta$ nirB M. tuberculosis (Fig. 1b) was compared to that of the wild-type in nitrogen-limiting medium, containing $1 \mathrm{mM}$ nitrite as the sole source of nitrogen. The mutant had lost its ability to assimilate nitrite. Cloning of nirBD from $M$. tuberculosis into the mutant restored the wild-type phenotype. Growth of $\Delta$ nirB $M$. tuberculosis was also tested in nitrogen-limiting medium with nitrate as the sole source of nitrogen. Again, no growth was detected (Fig. 1a). Thus, nirB encodes the assimilatory nitrite reductase of $M$. tuberculosis.

\section{Selection and characterization of mutants of $\boldsymbol{M}$. smegmatis defective in growth on nitrate}

An ethyl methane sulphonate (EMS) mutagenized $M$. smegmatis $\mathrm{mc}^{2} 155$ library was screened for mutants that were unable to grow on nitrogen-limiting agar, containing $10 \mathrm{mM}$ nitrate. We found two mutants, \#2009 and \#5192, that failed to grow on nitrate and nitrite, but showed robust growth on ammonium. We were able to complement these two mutants with an integrating cosmid library from M. tuberculosis for growth on nitrate (data not shown). Cosmids were isolated from complemented mutants, and sequence analysis showed that cosmids from both mutants had overlapping DNA fragments, including Rv0813c to Rv0839, corresponding to position 907338 to 936389 of the M. tuberculosis genome. Cosmids recovered from the mutant \#2009 also complemented the mutant $\# 5192$, and vice versa. These findings suggest that the mutations in \#2009 and \#5192 mapped to adjacent genes or the same gene. The complementing DNA fragment was narrowed down to Rv0818, which was able to rescue the $M$. smegmatis mutant \#2009 for growth on nitrate (Fig. 2). Mutant \#2009 grew less well on fully supplemented 7H10 medium. At present, the reason for this minimal growth defect remains unclear. It might be due to an unspecific effect as a result of the EMS mutagenesis in M. smegmatis, or to pleiotropic effects of the mutation. Rv0818 also complemented M. smegmatis mutant \#5192 (data not shown). A search of the TubercuList database (http:// genolist.pasteur.fr/TubercuList/) showed that Rv0818 shares $61.5 \%$ identity with the transcriptional regulator GlnR of S. coelicolor. GlnR is the global regulator of nitrogen assimilation in S. coelicolor.

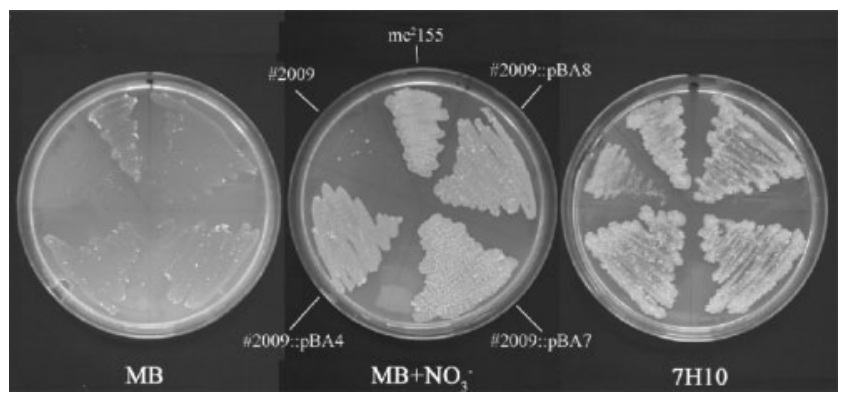

Fig. 2. Complementation of a M. smegmatis mutant for growth on nitrate. Strains were cultured on nitrogen-limiting agar (here referred to as $\mathrm{MB}$ ) containing $10 \mathrm{mM}$ nitrate $\left(\mathrm{MB}+\mathrm{NO}_{3}^{-}\right.$, middle), nitrogen-limiting agar without nitrate (MB, left), and rich $7 \mathrm{H} 10$ agar (7H10, right). Going from the top counterclockwise, the following strains were used: wild-type $M$. smegmatis $\mathrm{mc}^{2} 155$, the nitrate assimilation mutant of $M$. smegmatis (\#2009), the nitrate assimilation mutant of $M$. smegmatis \#2009 transformed with a chromosomal fragment from $M$. tuberculosis containing ORFs Rv0816c to Rv0820 (\#2009:: pBA4), and the nitrate assimilation mutant of $M$. smegmatis \#2009 transformed with ORF Rv0818 only (\#2009 : : pBA7, \#2009:: pBA8). 


\section{Growth of a $\Delta g I n R$ mutant of $M$. tuberculosis on nitrate}

We generated a $\Delta g \ln R$ (Rv0818) mutant in M. tuberculosis by introducing an unmarked 323 bp deletion within $g \ln R$ on the chromosome of $M$. tuberculosis. We compared growth of $\Delta g \ln R$ M. tuberculosis with that of the wild-type in nitrogen-limiting medium, with $10 \mathrm{mM}$ nitrate as the sole source of nitrogen. The $\Delta g \ln R$ mutant of $M$. tuberculosis was unable to grow on nitrate. Expression of $g \ln R$ on a plasmid in the $\Delta g \ln R$ mutant restored the wildtype phenotype (Fig. 3). Likewise, glnR from S. coelicolor also complemented $\Delta g \ln R M$. tuberculosis for growth on nitrate (Fig. 3).

The inability to reduce nitrite may result in the secretion of nitrite and rising levels in the medium. To elucidate the role of GlnR in nitrate assimilation of M. tuberculosis, we compared nitrite levels during culture of $M$. tuberculosis wild-type, $\Delta$ nirB M. tuberculosis and $\Delta g \ln R$ M. tuberculosis, as well as the complemented mutants. Strains were cultivated in nitrogen-limiting medium, with $10 \mathrm{mM}$ nitrate as the sole source of nitrogen. M. tuberculosis wild-type did not accumulate nitrite, because nitrate is reduced to nitrite by narGHJI-encoded nitrate reductase, and further reduced to ammonium by nirBD-encoded nitrite reductase. Both mutants, however, accumulated nitrite when cultured on nitrate (Fig. 4). To test whether the $g \ln R$ deletion affected the nitrate/nitrite assimilatory pathway beyond nitrite reduction, $\Delta g \ln R M$. tuberculosis was cultured on ammonium. The $\Delta g \ln R M$. tuberculosis was phenotypically indistinguishable from $M$. tuberculosis wild-type, as both strains utilized ammonium as a sole source of nitrogen (Fig. 3). Even though at this point we

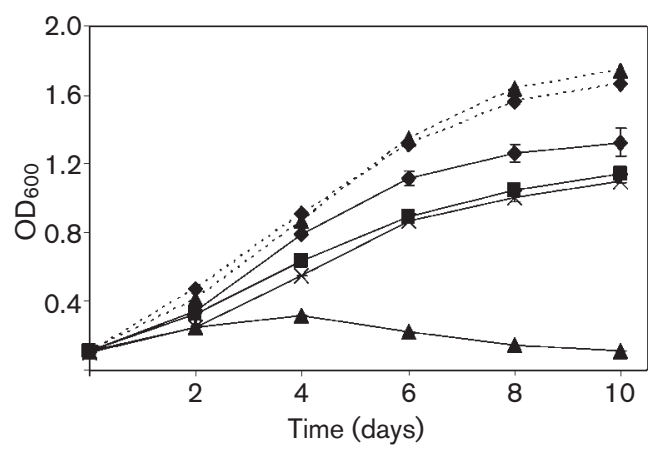

Fig. 3. Growth of $\Delta g \ln R$ mutant of $M$. tuberculosis on different sources of nitrogen. $M$. tuberculosis $\Delta g \ln R$ was cultured in nitrogen-limiting medium containing $10 \mathrm{mM}$ nitrate (continuous lines) or $10 \mathrm{mM}$ ammonium (dotted lines) as the only nitrogen source. Aliquots were tested for growth by measuring the $\mathrm{OD}_{600}$. Cultures were kept with good aeration. The following strains were compared: wild-type $M$. tuberculosis $(\boldsymbol{\nabla}), \Delta g \ln R$ M. tuberculosis (வ), $\quad \Delta g \ln R \quad$ M. tuberculosis:: $g \ln R_{\mathrm{MTB}}(\times), \quad \Delta g \ln R \quad M$. tuberculosis : : $g \ln R_{\mathrm{SCO}}(\boldsymbol{\square})$.

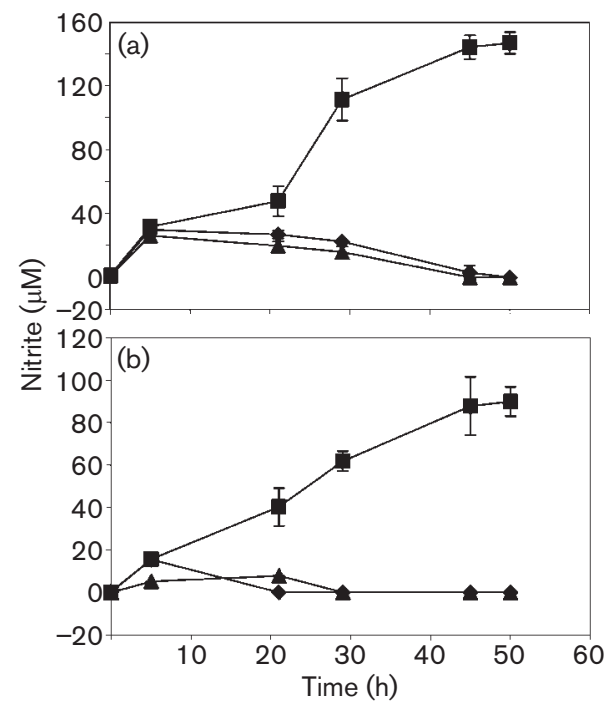

Fig. 4. Nitrite levels during culture of (a) $\Delta$ nirB M. tuberculosis and (b) $\Delta g \ln R M$. tuberculosis in nitrogen-limiting medium containing $10 \mathrm{mM}$ nitrate. At indicated time points aliquots were tested for accumulation of nitrite. The following strains were compared: (a) M. tuberculosis wild-type (A), M. tuberculosis carrying a deletion of $\operatorname{nir} B(\boldsymbol{\square}), M$. tuberculosis carrying a deletion of nirB transformed with nirB from $M$. tuberculosis ( $)$; (b) $M$. tuberculosis wild-type ( $\mathbf{\Delta}), M$. tuberculosis carrying a deletion of $g \ln R(\boldsymbol{\square}), M$. tuberculosis carrying a deletion of $g \ln R$ transformed with $g \ln R$ from M. tuberculosis $(\Delta)$.

could not exclude that the accumulation of nitrite might be caused also by other factors such as transport of nitrite, we hypothesized that the $\Delta g \ln R$ mutant reduces nitrate to nitrite, but is blocked in further reduction of nitrite to ammonium.

\section{Complementation of the M. tuberculosis $g / n R$ deletion mutant with nirBD}

In order to confirm that the lack of nitrate assimilation of the $g \ln R$ mutant is due to the lack of upregulation of nirBD in this mutant strain, we expressed nirBD under the transcriptional control of the constitutive hsp60 promoter in the $g \ln R$ mutant. We compared growth on nitrate as a sole source of nitrogen of the $g \ln R$ mutant strain and the mutant complemented with nirBD; growth of the wildtype in the same medium without nitrate was used as a control. The $g \ln R$ mutant strain was not able to grow on nitrate and behaved like the wild-type control cultured without nitrate (Fig. 5). The $g \ln R$ mutant strain complemented with nirBD showed an extended lag and acceleration phase, then replicated stably from day 4 until day 16 (Fig. 5). These results show that expression of $\operatorname{nir} B D$ allows the $g \ln R$ mutant to use nitrate as a sole source of nitrogen. 


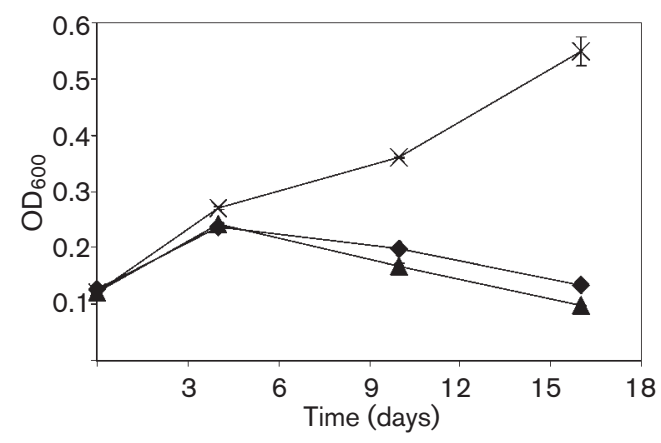

Fig. 5. Growth on nitrate of the glnR mutant of $M$. tuberculosis transformed with nirBD expressed from a heterologous promoter. Bacteria were cultured in nitrogen-limiting medium containing $10 \mathrm{mM}$ nitrate as the only nitrogen source. Aliquots were tested for growth by measuring the $\mathrm{OD}_{600}$. Cultures were kept with good aeration. The following strains were compared: $\Delta g \ln R \quad M$. tuberculosis ( $\mathbf{\Delta}), \Delta g \ln R$ M. tuberculosis : : nirBD under control of the heterologous hsp60 promoter $(\times)$, and the wild-type cultured without nitrogen as a control $(\nabla)$.

\section{GInR directly controls nirB expression}

Tiffert et al. (2008) showed that expression of nirB was lost in a $g \ln R$ mutant that had been generated by chemical mutagenesis of $S$. coelicolor. In the present study a putative GlnR-binding site within nirB of $M$. tuberculosis was analysed by performing an EMSA with purified GlnR of $M$. tuberculosis as well as purified GlnR of S. coelicolor. For purification of GlnR of $M$. tuberculosis, the glnR gene was fused to a StrepII-tag. An N-terminal Strep-glnR fusion gene could not be expressed in E. coli XL1 blue, as assessed by SDS-PAGE and Western blot analysis (data not shown). However, by using a C-terminal $g \ln R$-Strep construct, $g \ln R$ was successfully overexpressed and purified (data not shown). To investigate the ability of GlnR to bind to the nirB upstream region agarose EMSA was performed. Specific binding of GlnR of M. tuberculosis and S. coelicolor to the nirB upstream region of $M$. tuberculosis was observed (Fig. 6a, b).

To analyse the impact of $\mathrm{GlnR}$ on nirB expression, semiquantitative reverse transcription-PCR (RT-PCR) was performed, using wild-type M. tuberculosis H37Rv and the $\Delta g \ln R$ mutant strain. RNA was isolated from cultures that were grown in complex medium and subsequently transferred to nitrogen-limiting medium containing $5 \mathrm{mM} \mathrm{KNO}$ for $18 \mathrm{~h}$. Internal primers for the GlnR-regulated gene nirB were utilized (sigA was used as control, Fig. $6 \mathrm{c}$ ). In contrast to the parent strain of $M$. tuberculosis, expression of the nirB-encoded nitrite reductase was greatly reduced in the $\Delta g \ln R$ mutant (Fig. 6c).

To verify these results, we performed whole-genome expression profiling comparing the $g \ln R$ mutant of $M$. tuberculosis and the parent strain cultured in nitrogenlimiting medium with $5 \mathrm{mM}$ nitrate as the sole source of (a)

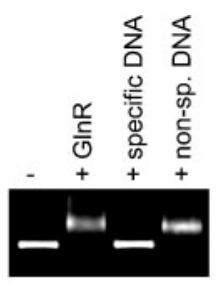

(c)

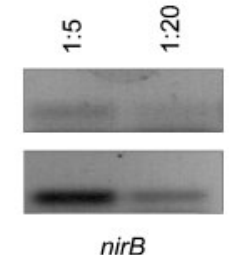

(b)

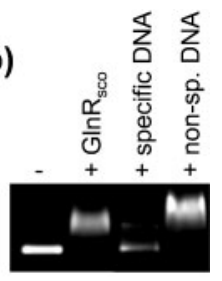

wild-type

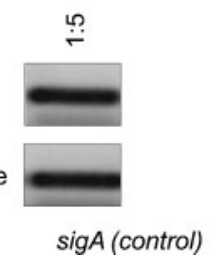

Fig. 6. GlnR regulates nir $B$ transcription. (a) Purified $G \ln R$ protein of M. tuberculosis was incubated with $2 \mathrm{ng}$ of $250 \mathrm{bp}$, Cy5labelled PCR fragments containing the upstream regions of nirB. The shifts were verified to be specific by adding 500 -fold excess of specific and non-specific DNA (non-labelled). (b) Purified $G \ln R_{\text {sco }}$ protein of $S$. coelicolor was incubated with $2 \mathrm{ng}$ of $250 \mathrm{bp}$, Cy5labelled PCR fragments containing the upstream regions of nirB. The shifts were verified to be specific by adding 500 -fold excess of specific and non-specific DNA (non-labelled). (c) RT-PCR analysis of GlnR target gene nirB in $M$. tuberculosis wild-type and $\Delta g / n R$ mutant. Two micrograms of RNA was used for reverse transcription. CDNA was diluted $1: 5$ and $1: 20$ and used for PCR analysis. $\operatorname{sig} A$ was used as control.

nitrogen as described above. For whole-genome expression profiling, we exposed bacilli to nitrogen-limiting medium providing $5 \mathrm{mM} \mathrm{KNO}$ for $18 \mathrm{~h}$ or $48 \mathrm{~h}$. Labelled cDNA from two independent experiments for each time point was subjected to array analysis. Two different time points from two independent experiments thus generated four datasets that were pooled and analysed. The results showed that nirB was upregulated 9.8 -fold $(P=0.003)$, and that nirD was upregulated 6.6 -fold $(P=0.017)$ in the wild-type strain compared with the $g \ln R$ mutant of $M$. tuberculosis. It is noteworthy that under the same conditions no difference in expression of narGHJI ( $\operatorname{narG}, 1.2$-fold, $P=0.639$; narH, 1.1 fold, $P=0.826$; narJ, 1.2 -fold, $P=0.437$; narI, 1.2 -fold, $P=0.599)$ was found between $M$. tuberculosis wild-type and the $g \ln R$ mutant.

\section{DISCUSSION}

When we started characterizing narGHJI of M. tuberculosis, we favoured the idea that NarGHJI is a purely anaerobic nitrate reductase (Weber et al., 2000). Sohaskey \& Wayne (2003) thoroughly studied expression of narGHJI of $M$. tuberculosis under various conditions. They found that the nitrate reductase activity of $M$. tuberculosis was sensitive to inhibition by both tungstate and azide, suggesting that the enzyme is a membrane-bound molybdenum-containing 
complex, which is typical for a respiratory enzyme. NarGHJI of $M$. tuberculosis also appeared to be functionally similar to that of E. coli, and complemented a narGHJIdefective strain of $E$. coli to support anaerobic growth (Sohaskey \& Wayne, 2003). These findings pointed to a potential role for this protein in anaerobic metabolism. However, the authors also reported that M. tuberculosis, unlike E. coli and B. subtilis, constitutively expressed narGHJI independent of the level of oxygen. Like $M$. tuberculosis, C. glutamicum and S. coelicolor belong to the order of Actinomycetales. C. glutamicum has one copy of narGHJI, whereas S. coelicolor has three copies of the gene cluster. In C. glutamicum, narGHJI serves as a respiratory nitrate reductase (Nishimura et al., 2007). The organism is not able to utilize nitrate as a sole source of nitrogen, suggesting the absence of nitrate assimilation (Nishimura et al., 2007; Takeno et al., 2007). In S. coelicolor the role of narGHJI is unclear. The organism utilizes nitrate as a sole source of nitrogen (Hodgson, 2000). However, it is not known whether this activity is mediated by narGHJI, and whether narGHJI encodes a respiratory nitrate reductase. Thus, at present $M$. tuberculosis appears to provide the first example of a narGHJI-encoded nitrate reductase that mediates assimilation of nitrate under aerobic conditions.

In E. coli, NirBD encodes an NADH-dependent nitrite reductase that is composed of two subunits. The enzyme is only synthesized during anaerobiosis, presumably to regenerate NAD and detoxify nitrite that accumulates as a result of nitrate respiration (Gennis \& Stewart, 1996). However, this is the exception to the rule, as most $\mathrm{NAD}(\mathrm{P}) \mathrm{H}$-dependent nitrite reductases consist of a single polypeptide, and have an assimilatory function (Lin \& Stewart, 1998). Here, we demonstrate that in M. tuberculosis NirBD functions as an assimilatory nitrate reductase. Homologues to nirBD of $M$. tuberculosis have been identified in S. coelicolor (SCO2486-SCO2488) but not in C. glutamicum (http://www.ncbi.nlm.nih.gov/sites/entrez). In the published literature, we found no direct evidence reporting reduction of nitrite in either species. However, as nitrate assimilation has been reported in S. coelicolor and requires formation of ammonium from nitrite, the homologues of nirBD might encode the assimilatory nitrite reductase in S. coelicolor (Tiffert et al., 2008).

In the present study, a $M$. tuberculosis $\Delta g \ln R$ mutant was also unable to grow on nitrate as the sole source of nitrogen. The $\Delta g \ln R$ mutant of $M$. tuberculosis grew on ammonium as well as the wild-type strain. One explanation for this observation is that GlnR controls the expression of narGHJI and/or nirB. We identified a GlnR-binding site upstream of nirB but not of narGHJI of M. tuberculosis. The effect of GlnR on the transcription of nirB was determined by semiquantitative RT-PCR. GlnR activates transcription of the nitrate-assimilatory gene nirB, which may explain the inability of the $\Delta g \ln R$ mutant to grow on nitrate as a sole source of nitrogen as well as the accumulation of nitrite. Whole-genome expression profiling of the $g \ln R$ mutant and the wild-type strain confirmed regulation of nirBD expression by GlnR, and showed that the regulator does not control expression of narGHJI. In $S$. coelicolor, it has been reported that GlnR also activates the expression of the nitrite reductase gene nirB (Tiffert et al., 2008). This and the successful complementation of the phenotype of the $M$. tuberculosis $\Delta g \ln R$ mutant with the $g \ln R$ gene of $S$. coelicolor provides further evidence that the role of NarGHJI, NirBD and GlnR in assimilation of nitrate is conserved between M. tuberculosis and S. coelicolor.

The signalling cascade for GlnR-mediated nirBD activation is unknown. Normally, OmpR-like regulators are specifically phosphorylated. OmpR itself is phosphorylated at a conserved aspartate residue (D-55) by the cognate sensor kinase EnvZ, resulting in a modulation of its DNA-binding affinity (Delgado et al., 1993). GlnR represents an orphan regulator with no coupled sensor kinase gene in the up- or downstream region. It is likely that GlnR is modified by an as yet unidentified kinase, as the protein contains the conserved aspartate residue (D-49) in its $\mathrm{N}$ terminus. A second gene, Rv2884, with homology to glnR of S. coelicolor, has been identified on the chromosome of M. tuberculosis. In S. coelicolor a second $g \ln R, g \ln R I I$, has been described (Fink et al., 2002). However, its inactivation did not produce a phenotype corresponding to that of a $S$. coelicolor $\Delta g \ln R$ mutant strain, pointing to GlnR as the principal regulator of nitrogen metabolism in S. coelicolor. Thus the role of Rv2884 in M. tuberculosis remains unclear at present.

In summary, this study describes the molecular mechanisms required for assimilatory nitrate and nitrite reduction, and its transcriptional control by GlnR in M. tuberculosis. Nitrate has been shown to accumulate in chronically infected tissue and might therefore be available to $M$. tuberculosis as a nutrient in the host. It might be utilized as an alternative substrate in cases of nitrogen limitation. In S. coelicolor GlnR has been suggested to play a global role in nitrogen metabolism (Tiffert et al., 2008). Further studies are ongoing that include additional genome expression profiling experiments, combined with confirmatory PCR and DNA-binding analysis, to address this issue in $M$. tuberculosis.

\section{ACKNOWLEDGEMENTS}

We thank S. Suerbaum for his support. This work was supported by the Niedersächsische Verein zur Bekämpfung der Tuberkulose, by the German Research Foundation (DFG) through the International Research Training Group 1273 to S.H., through grant SFB 587 to S.M., M.S. and F.-C.B., and by the state Lower Saxony through a Lichtenberg Fellowship to J. M. We thank J. D. McKinney for donation of the EMS-mutagenized M. smegmatis library. Y.T. acknowledges a scholarship from the Studienstiftung des deutschen Volkes. This work was supported by the DFG (SFB 587), EU (LSH 4032, ActinoGen) and by the BMBF as part of the SYSMO project (5019).

\section{REFERENCES}

Bange, F. C., Collins, F. M. \& Jacobs, W. R. (1999). Survival of mice infected with Mycobacterium smegmatis containing large DNA 
fragments from Mycobacterium tuberculosis. Tuber Lung Dis 79, 171180.

Beckers, G., Strosser, J., Hildebrandt, U., Kalinowski, J., Farwick, M., Kramer, R. \& Burkovski, A. (2005). Regulation of AmtR-controlled gene expression in Corynebacterium glutamicum: mechanism and characterization of the AmtR regulon. Mol Microbiol 58, 580-595.

Bogdan, C. (2001). Nitric oxide and the immune response. Nat Immunol 2, 907-916.

Burkovski, A. (2007). Nitrogen control in Corynebacterium glutamicum: proteins, mechanisms, signals. J Microbiol Biotechnol 17, 187194.

Cole, S. T., Brosch, R., Parkhill, J., Garnier, T., Churcher, C., Harris, D., Gordon, S. V., Eiglmeier, K., Gas, S. \& other authors (1998). Deciphering the biology of Mycobacterium tuberculosis from the complete genome sequence. Nature 393, 537-544.

Delgado, J., Forst, S., Harlocker, S. \& Inouye, M. (1993). Identification of a phosphorylation site and functional analysis of conserved aspartic acid residues of OmpR, a transcriptional activator for $o m p F$ and $o m p C$ in Escherichia coli. Mol Microbiol 10, 1037-1047.

DeTurk, W. E. \& Bernheim, F. (1958). Effects of ammonia, methylamine and hydroxylamine on the adaptive assimilation of nitrite and nitrate by a mycobacterium. J Bacteriol 75, 691-696.

Fink, D., Weissschuh, N., Reuther, J., Wohlleben, W. \& Engels, A. (2002). Two transcriptional regulators GlnR and GlnRII are involved in regulation of nitrogen metabolism in Streptomyces coelicolor A3(2). Mol Microbiol 46, 331-347.

Gennis, R. B. \& Stewart, V. (1996). Respiration. In Escherchia coli and Salmonella: Cellular and Molecular Biology, 2nd edn, pp. 217-261. Edited by F. C. Neidhardt and others. Washington, DC: America Society for Microbiology.

Hedgecock, L. W. \& Costello, R. L. (1962). Utilization of nitrate by pathogenic and saprophytic mycobacteria. J Bacteriol 84, 195-205.

Hodgson, D. A. (2000). Primary metabolism and its control in streptomycetes: a most unusual group of bacteria. Adv Microb Physiol 42, 47-238.

Lin, J. T. \& Stewart, V. (1998). Nitrate assimilation by bacteria. $A d v$ Microb Physiol 39, 1-30, 379.

McKinney, J. D., Höner zu Bentrup, K., Munoz-Elias, E. J., Miczak, A., Chen, B., Chan, W. T., Swenson, D., Sacchettini, J. C., Jacobs, W. R., Jr \& Russell, D. G. (2000). Persistence of Mycobacterium tuberculosis in macrophages and mice requires the glyoxylate shunt enzyme isocitrate lyase. Nature 406, 735-738.

Munoz-Elias, E. J. \& McKinney, J. D. (2006). Carbon metabolism of intracellular bacteria. Cell Microbiol 8, 10-22.

Nakano, M. M. \& Zuber, P. (1998). Anaerobic growth of a "strict aerobe" (Bacillus subtilis). Annu Rev Microbiol 52, 165-190.

Nishimura, T., Vertes, A. A., Shinoda, Y., Inui, M. \& Yukawa, H. (2007). Anaerobic growth of Corynebacterium glutamicum using nitrate as a terminal electron acceptor. Appl Microbiol Biotechnol 75, 889-897.

Ogawa, K., Akagawa, E., Yamane, K., Sun, Z. W., LaCelle, M., Zuber, P. \& Nakano, M. M. (1995). The nasB operon and nasA gene are required for nitrate/nitrite assimilation in Bacillus subtilis. $J$ Bacteriol 177, 1409-1413.

Pavelka, M. S. \& Jacobs, W. R. (1999). Comparison of the construction of unmarked deletion mutations in Mycobacterium smegmatis, Mycobacterium bovis bacillus Calmette-Guerin, and Mycobacterium tuberculosis $\mathrm{H} 37 \mathrm{Rv}$ by allelic exchange. J Bacteriol 181, 4780-4789.

Snapper, S. B., Melton, R. E., Mustafa, S., Kieser, T. \& Jacobs, W. R. (1990). Isolation and characterization of efficient plasmid transformation mutants of Mycobacterium smegmatis. Mol Microbiol 4, 1911-1919.

Sohaskey, C. D. \& Wayne, L. G. (2003). Role of narK2X and narGHJI in hypoxic upregulation of nitrate reduction by Mycobacterium tuberculosis. J Bacteriol 185, 7247-7256.

Stermann, M., Bohrssen, A., Diephaus, C., Maass, S. \& Bange, F. C. (2003). Polymorphic nucleotide within the promoter of nitrate reductase (NarGHJI) is specific for Mycobacterium tuberculosis. J Clin Microbiol 41, 3252-3259.

Stermann, M., Sedlacek, L., Maass, S. \& Bange, F. C. (2004). A promoter mutation causes differential nitrate reductase activity of Mycobacterium tuberculosis and Mycobacterium bovis. J Bacteriol 186, 2856-2861.

Stover, C. K., de la Cruz, V. F., Bansal, G. P., Hanson, M. S., Fuerst, T. R., Jacobs, W. R., Jr \& Bloom, B. R. (1992). Use of recombinant BCG as a vaccine delivery vehicle. Adv Exp Med Biol 327, 175-182.

Takeno, S., Ohnishi, J., Komatsu, T., Masaki, T., Sen, K. \& Ikeda, M. (2007). Anaerobic growth and potential for amino acid production by nitrate respiration in Corynebacterium glutamicum. Appl Microbiol Biotechnol 75, 1173-1182.

Tiffert, Y., Supra, P., Wurm, R., Wohlleben, W., Wagner, R. \& Reuther, J. (2008). The Streptomyces coelicolor GlnR regulon: identification of new GlnR targets and evidence for a central role of GlnR in nitrogen metabolism in actinomycetes. Mol Microbiol 67, 861-880.

Virtanen, S. (1960). A study of nitrate reduction by mycobacteria. The use of the nitrate reduction test in the identification of mycobacteria. Acta Tuberc Scand Suppl 48, 1-119.

Weber, I., Fritz, C., Ruttkowski, S., Kreft, A. \& Bange, F. C. (2000). Anaerobic nitrate reductase (narGHJI) activity of Mycobacterium bovis BCG in vitro and its contribution to virulence in immunodeficient mice. Mol Microbiol 35, 1017-1025.

Wray, L. V., Jr, Atkinson, M. R. \& Fisher, S. H. (1991). Identification and cloning of the $g \ln R$ locus, which is required for transcription of the glnA gene in Streptomyces coelicolor A3(2). J Bacteriol 173, 73517360 .

Edited by: G. R. Stewart 